\title{
Commonly diagnosed mental disorders in a general hospital system
}

\author{
George Scott ${ }^{1 *} \mathbb{C}$, Alessandra M. Beauchamp-Lebrón ${ }^{1}$, Ashley A. Rosa-Jiménez',
} Javier G. Hernández-Justiniano ${ }^{1,2}$, Axel Ramos-Lucca ${ }^{1,2}$, Gloria Asencio-Toro and Julio Jiménez-Chávez ${ }^{1,2^{*}}$

\begin{abstract}
Background: Considering many patients receive care from general hospitals, these healthcare institutions are uniquely situated to address mental and physical health needs. Little is documented, however, on the common current mental disorders diagnosed in patients receiving care in general hospital settings, especially in Puerto Rico. The objective of this study was to characterize the five most common current DSM-5 mental disorder diagnoses made in patients receiving non-psychiatric medical and surgical care from a general hospital system in southern Puerto Rico between January 2015 and December 2019.

Methods: Our clinical health psychology team provides integrated psychology consultation-liaison services to select clinical units in general hospitals across the southwestern region of Puerto Rico. The clinical team conducted routine standardized psychological evaluations at patients' bedside, arrived at a current DSM-5 diagnosis if warranted, and documented the diagnosis and other select variables. A retrospective study of cross-sectional data generated from the clinical team's standardized evaluations of 5494 medical patients was implemented. Multinomial logistic regression analyses were used to assess the odds of being diagnosed with a current DSM- 5 mental disorder during hospitalization.
\end{abstract}

Results: Overall, $53 \%$ of the entire sample was diagnosed with a mental disorder during hospitalization. Major depressive, neurocognitive, anxiety, substance-related and schizophrenia-spectrum disorders were the most frequently diagnosed. Interestingly, females were $23 \%$ less likely to have been diagnosed with major depressive disorder than males (aOR: $0.769, \mathrm{Cl}[0.650,0.909], \mathrm{p}=0.002$ ). This is to say males evidenced 1.30 higher odds of being diagnosed with depression compared to their female counterpart. Age, biological sex, civil status, employment status, monthly household income, previous mental disorder and history substance use/abuse history was differentially associated with receiving a current DSM-5 disorder.

Conclusion: The integration of clinical health psychology services within a general hospital facilitated our team's work of identifying and treating co-occurring mental disorders among hospitalized patients receiving medical and surgical care. Future studies examining the opportunities and barriers of integrating clinical health psychology services within a general hospital's administrative and clinical infrastructure for rapid identification and treatment of co-occurring mental disorders among medical patients is encouraged.

\footnotetext{
*Correspondence: gscott18@stu.psm.edu; jcjimenez@psm.edu

${ }^{1}$ Health Psychology Training and Research Core, School of Behavior

and Brain Sciences, Ponce Health Sciences University, 388 Zona Industrial

Reparada 2, Ponce, PR 00717, USA

Full list of author information is available at the end of the article
}

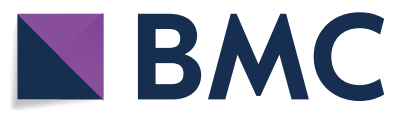

(c) The Author(s) 2021. This article is licensed under a Creative Commons Attribution 4.0 International License, which permits use, sharing, adaptation, distribution and reproduction in any medium or format, as long as you give appropriate credit to the original author(s) and the source, provide a link to the Creative Commons licence, and indicate if changes were made. The images or other third party material in this article are included in the article's Creative Commons licence, unless indicated otherwise in a credit line to the material. If material is not included in the article's Creative Commons licence and your intended use is not permitted by statutory regulation or exceeds the permitted use, you will need to obtain permission directly from the copyright holder. To view a copy of this licence, visit http://creativeco mmons.org/licenses/by/4.0/. The Creative Commons Public Domain Dedication waiver (http://creativecommons.org/publicdomain/ zero/1.0/) applies to the data made available in this article, unless otherwise stated in a credit line to the data. 
Keywords: Mental disorders, Depression, Neurocognitive disorder, General hospital, Health Psychology, Integrated care

\section{Introduction}

Mental disorders are among the top causes of morbidity and account for approximately $14 \%$ of all deaths globally [45] and approximately $11 \%$ of the world's population currently lives with a mental disorder [29]. Alarmingly, this estimate is higher in Puerto Rico, as revealed by findings from a recent behavioral health needs assessment conducted on the island. This assessment reported 12-month prevalence rates for any mental disorder to be approximately $24 \%$ among adults ages 18 to 64 [9]. Moreover, Puerto Ricans with a serious mental illness make up $7.3 \%$ of the adult population. It is estimated that nearly half (43.1\%) of Puerto Rican adults with some type of mental disorder are in need of mental healthcare services [9]. These findings elucidate the magnitude of the mental health disparity crisis on the island, with inaccessible quality mental healthcare being a leading driver of such disparity [21, 43]. Since 2008, there has been a consistent migratory stream from the island to the United States. Included in this efflux of people are specialty mental healthcare providers such as clinical psychologists and psychiatrists, which further worsens the conundrum of lack of access to quality mental healthcare services [41, 42]. Thus, there is an urgent need to address this. Considering many patients receive medical and surgical care from general hospitals, these institutions are uniquely situated to facilitate access to mental healthcare services through rapid identification of mental disorders and initiation of appropriate interventions. In fact, a recent meta-analysis revealed that between 4 and $32 \%$ of inpatients receiving care in a non-psychiatric general hospital setting evidenced clinically significant levels of depression [46]. An earlier review of epidemiological studies revealed the prevalence of mental disorders in general hospital inpatients ranged from 41 to $46 \%$, with organic brain syndrome, adjustment disorders with depressed mood and alcohol abuse being the three most commonly diagnosed [30]. Such a characterization has yet to be accomplished in Puerto Rico. Thus, the current study's research team implemented a retrospective observational study of cross-sectional data to assess the most frequently diagnosed mental disorders in medical and surgical inpatients of a general hospital system in southern Puerto Rico between 2015 and 2019.

In order to achieve such a characterization, the systems that facilitate rapid identification of mental disorders and the provision of mental health services for medical and surgical inpatients of a general hospital are of critical value and worth briefly mentioning. Two widely used systems to integrate mental and physical health care of medical patients are the collaborative care [40] and consultation-liaison models [39]. The collaborative care model is mostly used in outpatient, primary-care settings and have limited implications for use in general hospital settings. The consultation-liaison (C-L) model, however, is more relevant for this present study's aim and in fact, is the model which was used by our clinical team as detailed in the methods section below. Briefly, the C-L model entails two distinct yet overleaping components as the name implies: consultation and liaison. The consultation component is a process whereby attending physicians, surgeons, patients themselves or family members may request mental health services, and a clinical team member responds to this request by conducting an initial assessment, arriving at a clinical diagnostic impression, facilitating interventions as needed and making appropriate referrals if warranted. Liaison services are an especially crucial aspect in the provision of mental health care services to medical inpatients as this component requires a certain degree of integration at not only the service level (i.e., multidisciplinary teams) but at the organizational level as well [37]. Most attention in the literature, however, is given to the consultation aspect of the C-L model [14]. This selective attention is rather unfortunate as the liaison component, as overviewed in the discussion section below, is an aspect that warrants urgent attention and widespread implementation to achieve integrated care models in general hospital settings in an effort to curtail the mental health disparities that exist among medical populations such as in Puerto Rico. The success of detecting current mental disorders and facilitating interventions among the sample of medical and surgical inpatients of this present study is owed, in part, to the clinical health psychology $\mathrm{C}$ - $\mathrm{L}$ integration model implemented.

\section{Methods}

The research team conducted a retrospective study examining cross-sectional biopsychosocial variables obtained from a sample of 5494 inpatients that received medical or surgical care from a general hospital in Ponce, Puerto Rico between January 2015 and December 2019.

\section{Participants}

Participants were inpatients age 21 years and older, admitted to cardiovascular surgery, coronary care, 
intensive care and skilled nursing units of Damas Hospital, a general hospital in Ponce, Puerto Rico. These patients received a standardized clinical evaluation at bedside during their hospital stay and gave informed consent for data collection. Patients diagnosed with a current depressive, neurocognitive, anxiety, substancerelated, and addictive and schizophrenia spectrum disorders according to DSM-5 criteria during their bedside standardized clinical evaluation were included in the study as these diagnostic categories were the most commonly diagnosed during this four-year period. Additionally, those who did not meet DSM-5 criteria for a current diagnosis during hospitalization were included as a comparison group. The Institutional Review Board of the Ponce Research Institute approved this study. Every author certifies that the study was performed in accordance with the ethical standards, as set by the 1964 Declaration of Helsinki and its later amendments.

\section{Procedures \\ Clinical team}

The Clinical Psychology Services Program (CPSP) of Ponce Health Sciences University provides clinical health psychology services that include screenings, standardized clinical evaluations, brief psychotherapy, psychophysiological interventions, neuropsychological rehabilitation, and consultation-liaison (C-L) services in general hospital settings across the southern region of Puerto Rico. The provision of $\mathrm{C}$ - $\mathrm{L}$ health psychology services at Damas Hospital is the product of an academic agreement between Ponce Health Sciences University and Damas Hospital, an agreement that has been ongoing since 2002. The clinical team is coordinated by a psychiatrist and is made up of three licensed clinical psychologists, two of which are health psychologists, and one is a certified neuropsychologist. Additionally, 12 advanced practicum students of the PhD and PsyD clinical psychology programs of Ponce Health Sciences University are part of the clinical team and receive on-site, same day supervision for each patient seen and standardized clinical interview performed. The CPSP have established agreements with affiliate hospitals where standing orders are in place for select hospital units. Standing orders are the backbone of the clinical team's liaison services and entail the administration of routine standardized clinical evaluations for all patients admitted to the following units: (a) cardiovascular surgery, (b) coronary care, (c) intensive care, and (d) skilled nursing units. Additionally, the clinical team provided clinical health psychology consults as requested by attending physicians, regardless of the inpatient unit to which the patient was admitted. For this present study, the research team analyzed sociodemographic and psychosocial data, current ICD-10 medical diagnoses, and current DSM-5 mental disorder diagnoses the CPSP clinical team made at patients' bedside during the standardized clinical evaluation.

\section{Standardized clinical evaluation}

The standardized clinical evaluation entailed a mental status examination, exploration of psychological and emotional symptoms, a review of past physical and mental illness history, and a review of social functioning. Clinical inventories such as the Mini Mental Status Exam [15], Beck Depression Inventroy-II [6], and Beck Anxiety Inventory [7] were used to aid in the exploration of mental status and psychological-emotional symptomology. Additionally, a review of laboratory results, medications, and physician and nurse progress notes was conducted to aid in ruling out organic causes of possible mental aberrations. The CPSP clinical team performed the standardized clinical evaluation at the patient's bedside. If warranted, the clinical team made a current DSM-5 diagnosis and a corresponding brief treatment plan was implemented for the duration of the patient's hospital stay. For patients that did not evidence clinically significant symptoms as determined by the DSM- 5 during evaluation, no diagnosis was made, and these patients' data was used as a comparison group. The clinical inventories mentioned above were not included in the data analysis process as these measures were not available in the digital database alongside the current DSM- 5 diagnoses and sociodemographic variables.

\section{Measures \\ Sociodemographic, medical diagnosis, and past mental health history}

Questionnaires generated by the clinical team were used to collect the following biopsychosocial variables: age, biological sex, civil status, level of education, employment status, and monthly household income. Past history of mental disorder and history of substance use/abuse variables were also included in the study. ICD-10 medical diagnoses were obtained from chart reviews performed by the CPSP clinical team.

\section{Data analysis}

SPSS 27 (IBM Corp) was used to perform data analysis. Data preparation and exploration procedures were conducted, and data was examined for errors and quality. Missing data analysis was conducted, and missing variables were replaced with multiple imputation linear regression method, with 10 imputations utilized. [31]. All missing data $(12-$ monthly household income variables and 23-civil status variables) in this dataset were less than 5\%. Descriptive statistics were performed to assess measures of central tendencies, standard deviation, and 
confidence intervals. Chi-squared tests of independence were conducted to assess associations between the top five diagnosed mental disorders and select sociodemographic indicators. Multinomial logistic regressions were conducted to evaluate the odds of being diagnosed with a DSM-5 depressive, neurocognitive, anxiety, substancerelated, or schizophrenia spectrum disorder compared to no disorder among inpatients. The Benjamini-Hockberg procedure [8] was applied to control for false discovery rates due to multiple comparisons in the adjusted multinomial logistic regression model.

\section{Results}

Data from a total of 5494 inpatients was analyzed. The mean age of this patient sample was 62.2 years, $95 \% \mathrm{CI}$ $[61.75,62.65]$. There were more females $(57.8 \%)$ than males and the majority of patients were unmarried (53.2\%). Additionally, 83.6\% were unemployed or retired and $26 \%$ reported having obtained less than a high school education. Overall, more than half $(53 \%)$ of the entire sample was diagnosed with a current DSM-5 mental disorder during hospitalization. Major depressive disorders accounted for $42 \%$, neurocognitive (33\%), anxiety (11\%), substance-related (8\%), and schizophrenia spectrum (6\%) of the five most frequently diagnoses made by the CPSP clinical team. Further, $97 \%$ of all patients evidenced a chronic physical condition. Ninety-nine per cent (99.4\%) of male patients had a chronic physical condition compared to $95 \%$ of female patients. Fifty-nine percent (59\%) of male patients had comorbid mental and physical conditions compared to $48 \%$ of females.

\section{Specific DSM-5 diagnoses}

Among major depressive disorders diagnoses (MDD), recurrent episodes (70\%) were most frequently diagnosed and recurrent episodes with psychotic characteristics (6.9\%), the least frequent. Within neurocognitive disorders (NCD), delirium due to another medical condition (24.8\%) was most commonly diagnosed and unspecified delirium (6.8\%) the least common. Generalized anxiety disorder (72.5\%) was the most frequently diagnosed anxiety disorder and alcohol use disorder (48\%) the most frequently diagnosed substance-related disorder. Schizophrenia and brief psychotic disorder were the most and least common diagnosed within the schizophrenia spectrum disorders, $34.6 \%$ and $6.7 \%$, respectively.

\section{Comorbid chronic physical conditions and diagnosed mental disorders}

Overall, cardiovascular disease, orthopedic conditions, chronic respiratory, gastrointestinal and cancer diagnoses were the most prevalent comorbid medical conditions in this sample. Thirty-five percent (35\%) of this sample were evaluated in the skilled nursing unit, $24 \%$ coronary care, $22 \%$ intensive care and 19\% cardiovascular surgery unit. Among patients diagnosed with major depressive disorders, slightly more than one in five patients (24\%) also lived with a co-occurring cardiovascular disease. The least frequent co-occurring chronic physical disease among patients with depressive disorders was immunologic disease, accounting for $0.2 \%$ of all ICD-10 diagnoses among this patient group. Chronic neurological diseases were the most common co-occurring physical conditions among patients diagnosed with a DSM-5 neurocognitive condition $(22 \%)$, and chronic endocrine disorders (1\%) were the least common. Slightly more than one out of every four patients (27\%) diagnosed with an anxiety disorder also had a co-occurring cardiovascular disease. Chronic immunologic disorders were least common among this group of patients, accounting for $0.3 \%$ of ICD-10 diagnoses. Similarly, patients diagnosed with a substance-related disorder had higher cardiovascular disease comorbidity (18\%) than any other chronic physical condition. Lastly, among patients diagnosed with a schizophrenia spectrum disorder, $5.8 \%$ also lived with a co-occurring cardiovascular disease.

Routine standardized clinical evaluations as part of the administrative standing orders accounted for $84 \%$ of all DSM-5 diagnoses made and the remaining $16 \%$ were made from physician or patient requested consults. The two most common diagnoses resulting from the administrative standing orders (routine standardized clinical interviews-the liaison component) were neurocognitive and major depressive disorders, accounting for $20 \%$ and $19 \%$, respectively. Diagnoses made from all consults, however, consisted primarily of major depressive disorders $(37 \%)$, followed by substance use disorders (9\%).

\section{Association of biological sex and mental disorder diagnoses in a general hospital setting}

An unadjusted multinomial logistic regression model revealed that biological sex is differentially associated with being diagnosed with a current mental disorder during hospitalization. The overall model was significant $\chi^{2}$ $(5)=215.659, \mathrm{p}<0.001$, and biological sex accounted for $4.1 \%$ of the variance found within the mental disorder diagnosis categories (Nagelkerke pseudo $R^{2}=0.041$ ). Overall, male patients had higher odds of being diagnosed with a current DSM-5 diagnosis compared to females. However, being diagnosed with an anxiety disorder was not significantly associated with biological sex. Female patients were $15.7 \%$ less likely to be diagnosed with MDD compared to male (OR: 0.842 , $95 \%$ CI [0.732, 0.968 ], $\mathrm{p}=0.01$ ). This is to say males were 1.18 times more likely to have been diagnosed with a current MDD at bedside than females. Moreover, males evidenced 1.78 
higher odds of being diagnosed with a form of NCD compared to females (OR: 0.561, CI [0.484, 0.651], p<0.001). Male patients also evidenced much higher odds (7.46) of being diagnosed with a substance related disorder compared to females (OR: 0.134, CI [0.094, 0.190], $\mathrm{p}<0.001$ ). Lastly, female patients were $49.5 \%$ less likely to be diagnosed with a schizophrenia spectrum disorder than males (OR: 0.505, CI [0.371, 0.688], p<0.001). Stated differently, male patients evidenced 1.98 higher odds of being diagnosed with a schizophrenia spectrum disorder during hospitalization see Tables 1, 2, 3 .

\section{Adjusted multinomial model}

The overall adjusted model was significant $X^{2}$ $(70)=2463.171, \quad \mathrm{p}<0.001$, with covariate variables accounting for $38.5 \%$ of the variance found within the DSM-5 diagnosis categories (Nagelkerke pseudo

Table 1 Sociodemographic Characteristics $(n=5494)$

\begin{tabular}{|c|c|c|}
\hline Variable & Frequency & $\%$ \\
\hline \multicolumn{3}{|l|}{ Age group (years) } \\
\hline $21-30$ & 425 & 7.7 \\
\hline $31-40$ & 342 & 6.2 \\
\hline $41-50$ & 441 & 8 \\
\hline $51-60$ & 923 & 16.8 \\
\hline $61-70$ & 1416 & 25.8 \\
\hline $71-80$ & 1286 & 23.4 \\
\hline $81-90$ & 568 & 10.3 \\
\hline$\geq 91$ & 93 & 1.7 \\
\hline \multicolumn{3}{|l|}{ Biological sex } \\
\hline Female & 3175 & 57.8 \\
\hline Male & 2319 & 42.2 \\
\hline \multicolumn{3}{|l|}{ Civil status } \\
\hline Not married & 2924 & 53.2 \\
\hline Married & 2570 & 46.8 \\
\hline \multicolumn{3}{|l|}{ Employment status } \\
\hline Not employed (including retired) & 4593 & 83.6 \\
\hline Employed & 901 & 16.4 \\
\hline \multicolumn{3}{|l|}{ Education level } \\
\hline No formal education & 93 & 1.7 \\
\hline$<$ High school & 1348 & 24.5 \\
\hline High school & 2181 & 39.7 \\
\hline Postsecondary and beyond & 1872 & 34.1 \\
\hline \multicolumn{3}{|l|}{ Monthly household income (USD) } \\
\hline$\$ 0$ & 120 & 2.2 \\
\hline$\leq \$ 300$ & 612 & 11.1 \\
\hline$\$ 301-\$ 900$ & 2091 & 38.1 \\
\hline$\$ 901-\$ 1500$ & 1457 & 26.5 \\
\hline$\$ 1501-\$ 2100$ & 602 & 11 \\
\hline$\geq \$ 2101$ & 612 & 11.1 \\
\hline
\end{tabular}

$\mathrm{R}^{2}=0.385$ ). Reference Table 4 for adjusted multinomial logistic regression values.

\section{Major depressive disorders}

Overall, age, biological sex, civil status, employment status, monthly household income, previous mental disorder and substance use/abuse history was significantly associated with receiving a current DSM-5 major depressive disorder diagnosis. For each one-year increase in age, patients evidenced a $1 \%$ less chance of being diagnosed with a current MDD (OR: 0.990, CI [0.985, 0.995]). Females evidenced 23\% lower odds of being diagnosed compared to males (OR: 0.769, CI [0.650, 0.909]). In other words, males had 1.30 higher odds of being diagnosed with a current depressive disorder. Compared to married patients, the ones who reported being unmarried were 1.41 times more likely to have been diagnosed (OR: 1.41 , CI $[1.20,1.65])$. Those who reported having a monthly household income of $\$ \leq 300$ had 2.12 higher odds of being diagnosed compared to patients reporting a household income $\geq \$ 2101$ per month (OR: 2.12 , CI $[1.53,2.94])$. Patients with previous mental disorders evidenced 7.62 higher odds of being diagnosed with a current depressive disorder compared to patients without a previously diagnosed mental disorder (OR: 7.62, CI [6.50, 8.95]). Lastly, patients with a past history of substance use/abuse evidenced 1.23 higher odds of being diagnosed with depression compared to those with no history of substance use/abuse (OR: 1.23, CI [1.03, 1.48]). Level of education was not significantly associated with being diagnosed with a current depressive disorder diagnosis at bedside. After adjusting for multiple comparisons, only age, biological sex, civil status, employment status, monthly household incomes of $\leq \$ 300$, and previous mental disorder remained significant covariates of being diagnosed with a current MDD.

\section{Neurocognitive disorders}

For every one-year increase in age, the odds of being diagnosed with a current neurocognitive disorder was relatively similar (OR: 1.058, CI [1.051, 1.065]). Female patients evidenced $45 \%$ lower odds of being diagnosed compared to males (OR; 0.54, CI [0.46, 0.64]). This is to say males had 1.82 higher odds than females of being diagnosed with a current neurocognitive disorder. Unmarried patients were 1.27 times more likely to have received a current diagnosis compared to married patients (OR: 1.27, CI [1.08, 1.49]). Those who reported a monthly household income $\leq \$ 300$ evidenced 1.75 higher odds of being diagnosed compared to patients with incomes $\geq \$ 2101$ per month (OR: 1.75, CI [1.25, 2.46]). Patients with a previous mental disorder had 1.90 higher odds of receiving a neurocognitive diagnosis compared 
Table 2 Profile of sociodemographic, previous mental disorder history and chronic co-morbid physical illness

\begin{tabular}{|c|c|c|c|c|c|c|}
\hline$(n=5494)$ & $\begin{array}{l}\text { Major depressive } \\
\text { disorder } \\
(n=1214)\end{array}$ & $\begin{array}{l}\text { Neurocognitive } \\
(n=995)\end{array}$ & Anxiety $(n=314)$ & $\begin{array}{l}\text { Substance-related } \\
(\mathrm{n}=217)\end{array}$ & $\begin{array}{l}\text { Schizophrenia } \\
\text { spectrum } \\
(n=173)\end{array}$ & $\begin{array}{l}\text { No DSM-5 } \\
\text { diagnosis } \\
(\mathrm{n}=2581)\end{array}$ \\
\hline \multicolumn{7}{|l|}{ Mean $(95 \% \mathrm{Cl})$} \\
\hline Age (years) & $57.63(56.68,58.58)$ & $73.41(72.64,74.17)$ & $56.90(54.94,58.86)$ & $51.71(49.45,53.97)$ & $49.83(47.23,52.64)$ & $62.30(61.66,62.94$ \\
\hline \multicolumn{7}{|c|}{ Frequency (\% within mental disorder category) } \\
\hline \multicolumn{7}{|l|}{ Biological sex } \\
\hline Female & $722(59.5)$ & $492(49.4)$ & $199(63.4)$ & $41(18.9)$ & $81(46.8)$ & $1640(63.5)$ \\
\hline Male & $492(40.5)$ & $503(50.6)$ & $115(36.6)$ & $176(81.1)$ & $92(53.2)$ & $941(36.5)$ \\
\hline \multicolumn{7}{|l|}{ Civil status } \\
\hline Not married & $734(60.5)$ & $521(52.4)$ & $164(52.2)$ & $153(70.5)$ & $135(78.0)$ & $1217(47.2)$ \\
\hline Married & $480(39.5)$ & $474(47.6)$ & $150(47.8)$ & $64(29.5)$ & $38(22.0)$ & $1364(52.8)$ \\
\hline \multicolumn{7}{|l|}{ Education level } \\
\hline $\begin{array}{l}\text { No formal educa- } \\
\text { tion }\end{array}$ & $17(1.4)$ & $27(2.7)$ & $4(1.3)$ & $2(0.9)$ & $2(1.2)$ & $41(1.6)$ \\
\hline $\begin{array}{l}\text { Less than high } \\
\text { school }\end{array}$ & $268(22.1)$ & $323(32.5)$ & $62(19.7)$ & $55(25.3)$ & $48(27.7)$ & $592(22.9)$ \\
\hline High school & $508(41.8)$ & $375(37.7)$ & $123(39.2)$ & $111(51.2)$ & $81(46.8)$ & $983(38.1)$ \\
\hline $\begin{array}{l}\text { Postsecondary } \\
\text { and beyond }\end{array}$ & $421(34.7)$ & $270(27.1)$ & $125(39.8)$ & $49(22.6)$ & $42(24.3)$ & $965(37.4)$ \\
\hline \multicolumn{7}{|l|}{ Employment status } \\
\hline Not employed & $1044(86.0)$ & $921(92.6)$ & $234(74.5)$ & $168(77.4)$ & $159(91.9)$ & $2067(80.1)$ \\
\hline Employed & $170(14.0)$ & $74(7.4)$ & $80(25.5)$ & $49(22.6)$ & $14(8.1)$ & $514(19.9)$ \\
\hline \multicolumn{7}{|c|}{ Monthly household income } \\
\hline$\leq \$ 300$ & $221(18.2)$ & $130(13.1)$ & $29(9.2)$ & $54(24.9)$ & $48(27.7)$ & $250(9.7)$ \\
\hline$\$ 301-\$ 900$ & $462(38.1)$ & $403(40.5)$ & $131(41.7)$ & $82(37.8)$ & $83(48.0)$ & $930(36.0)$ \\
\hline$\$ 901-\$ 1500$ & $310(25.5)$ & $269(27.0)$ & $79(25.2)$ & $46(21.2)$ & $27(15.6)$ & $726(28.1)$ \\
\hline$\$ 1501-\$ 2100$ & $107(8.8)$ & $96(9.6)$ & $35(11.1)$ & $20(9.2)$ & $8(4.6)$ & $336(13.0)$ \\
\hline$\geq \$ 2100$ & $114(9.4)$ & $97(9.7)$ & $40(12.7)$ & $15(6.9)$ & $7(4.0)$ & $339(13.1)$ \\
\hline \multicolumn{7}{|c|}{ History of substance use/abuse } \\
\hline Positive history & $391(32.2)$ & $238(23.9)$ & $87(27.7)$ & $196(90.3)$ & $68(39.3)$ & $568(22.0)$ \\
\hline Negative history & $823(67.8)$ & $757(76.1)$ & $227(72.3)$ & $21(9.7)$ & $105(60.7)$ & $2013(78.0)$ \\
\hline \multicolumn{7}{|c|}{ Previous mental disorder } \\
\hline $\begin{array}{l}\text { Recurring mental } \\
\text { disorder }\end{array}$ & 774 (63.8) & $225(22.6)$ & $159(50.6)$ & $102(47.0)$ & $152(87.9)$ & $459(17.8)$ \\
\hline $\begin{array}{l}\text { No recurring } \\
\text { mental dis- } \\
\text { order }\end{array}$ & $440(36.2)$ & $770(77.4)$ & $155(49.4)$ & $115(53.0)$ & $21(12.1)$ & $2122(82.2)$ \\
\hline \multicolumn{7}{|c|}{ Chronic co-morbid physical illness } \\
\hline $\begin{array}{l}\text { Cardiovascular } \\
\text { Disease }\end{array}$ & $291(24.0)$ & $198(19.9)$ & $84(26.8)$ & $40(18.4)$ & $10(5.8)$ & $689(26.7)$ \\
\hline Orthopedic & $77(6.3)$ & $110(11.1)$ & $34(10.8)$ & $16(7.4)$ & $1(0.6)$ & $485(18.8)$ \\
\hline Respiratory & $96(7.9)$ & $110(11.1)$ & $34(10.8)$ & $9(4.1)$ & $10(5.8)$ & $123(4.8)$ \\
\hline Gastrointestinal & $74(6.1)$ & $76(7.6)$ & $22(7.0)$ & $17(7.8)$ & $8(4.6)$ & $155(6.0)$ \\
\hline $\begin{array}{l}\text { Cancer (unspeci- } \\
\text { fied) }\end{array}$ & $38(3.1)$ & $17(1.7)$ & $10(3.2)$ & $6(2.8)$ & $2(1.2)$ & $146(5.7)$ \\
\hline Other & $632(52.1)$ & $482(48.4)$ & $129(41.1)$ & $128(59.0)$ & $142(82.0)$ & $830(32.2)$ \\
\hline No diagnosis & $6(0.5)$ & $2(0.2)$ & $1(0.3)$ & $1(0.5)$ & 0 & $153(5.9)$ \\
\hline
\end{tabular}

to patients without a previous illness (OR: 1.90, CI [1.57, 2.31]). Employment status, level of education, and history of substance use/abuse was not significantly associated with being diagnosed with a current DSM-5 neurocognitive disorder. Further, after adjusting for multiple comparisons, all sociodemographic and previous mental disorders remained significant. 
Table 3 Specific DSM-5 diagnoses, consultation and liaison services

\begin{tabular}{|c|c|c|}
\hline & Frequency & $\begin{array}{l}\text { \% (within } \\
\text { DSM-5 } \\
\text { category) }\end{array}$ \\
\hline \multicolumn{3}{|l|}{ Major depressive disorders (MDD) $(n=1214)$} \\
\hline MDD, recurrent episode & 855 & 70.4 \\
\hline MDD, single episode & 122 & 10.1 \\
\hline $\begin{array}{l}\text { MDD, recurrent with psychotic character- } \\
\text { istics }\end{array}$ & 84 & 6.9 \\
\hline Other & 153 & 12.6 \\
\hline \multicolumn{3}{|l|}{ Neurocognitive disorders (NCD) $(n=995)$} \\
\hline Delirium due to another medical condition & 247 & 24.7 \\
\hline Unspecified NCD & 148 & 14.8 \\
\hline Delirium due to multiple etiologies & 92 & 9.3 \\
\hline Mild NCD due to vascular disease & 80 & 8.0 \\
\hline Unspecified delirium & 68 & 6.8 \\
\hline Other & 360 & 36.2 \\
\hline \multicolumn{3}{|l|}{ Anxiety disorders $(n=314)$} \\
\hline Generalized anxiety disorder (GAD) & 228 & 72.5 \\
\hline Panic disorder & 54 & 17.2 \\
\hline Other & 32 & 10.1 \\
\hline \multicolumn{3}{|l|}{ Substance-related $(n=217)$} \\
\hline Alcohol use disorder & 104 & 48.0 \\
\hline Tobacco use disorder & 93 & 42.8 \\
\hline Other & 20 & 9.2 \\
\hline \multicolumn{3}{|l|}{ Schizophrenia spectrum $(n=173)$} \\
\hline Schizophrenia & 60 & 34.6 \\
\hline $\begin{array}{l}\text { Unspecified schizophrenia spectrum and } \\
\text { other psychotic disorder }\end{array}$ & 36 & 20.7 \\
\hline Brief psychotic disorder & 11 & 6.7 \\
\hline Other & 66 & 38.0 \\
\hline \multicolumn{3}{|l|}{ Type of service } \\
\hline Consultation & 856 & 16 \\
\hline Standing order & 4638 & 84 \\
\hline
\end{tabular}

"other" diagnostic category was commonly diagnosed in this sample to warrant mentioning

\section{Anxiety disorders}

Only age and recurring illness was significantly associated with being diagnosed with a current anxiety condition. For every one-year increase in age, patients had $0.2 \%$ lower odds of being diagnosed (OR: 0.989, CI [0.981, 0.997]). Having a previous mental disorder was related to 4.46 higher odds of receiving a current diagnosis (OR: 4.46, CI [3.47, 5.72]). Both age and previous mental disorder remained significantly linked to a current anxiety disorder diagnosis after adjusting for multiple comparisons.

\section{Substance-related disorders}

Each year increase in age was associated with $2.9 \%$ lower odds of being diagnosed with a current substance-related disorder (OR: 0.97, CI [0.96, 0.98]). Female patients evidenced $73.2 \%$ lower odds of being diagnosed compared to males (OR: 0.26, CI [0.18, $0.39]$ ). Conversely, males evidenced 3.73 higher odds of being diagnosed with a substance-related disorder. Unmarried patients evidenced 1.90 higher odds of receiving a diagnosis compared to married patients (OR: $1.90, \mathrm{CI}[1.34,2.68])$. Those who reported monthly household incomes of $\leq \$ 300$ were 2.76 times more likely to have been diagnosed compared to those with a monthly income of $\geq \$ 2101$ (OR: $2.76, \mathrm{CI}[1.40,5.44]$ ). Having a previous mental disorder was associated with 2.86 higher odds of receiving a current diagnosis compared to not having a previous illness (OR: 2.86, CI $[2.06,3.97])$. Lastly, those with a history of substance use/abuse were 16.98 times more likely to have been diagnosed with a current substance-related disorder compared to patients without substance use/abuse history (OR: 16.98, CI [10.48, 27.49]). Level of education and employment were not significantly associated with receiving a current DSM-5 substance-related diagnosis. All covariates remained significant after adjusting for multiple comparisons.

\section{Schizophrenia spectrum disorders}

Each one-year increase in age was associated with $2.8 \%$ less odds of being diagnosed with a current schizophrenia spectrum disorder (OR: 0.97, CI [0.96, 0.98]). Female patients were $60 \%$ less likely to have been diagnosed compared to males (OR: 0.39 , CI $[0.27,0.56])$. This is to say male patients evidenced 2.53 higher odds of being diagnosed with a schizophrenia spectrum disorder compared to females. Unmarried patients were 2.49 odds more likely to have been diagnosed compared to married patients (OR: 2.49, CI [1.66, 3.73]). Those with less than high school education evidenced 2.13 higher odds of being diagnosed (OR: 2.13, CI [1.31, 3.47]) compared to patients with postsecondary education attainment or beyond. Patients with monthly household incomes of $\leq \$ 300$ and $\$ 301-\$ 900$ had 4.28 and 3.05 higher odds, respectively, of being diagnosed compared to those with household incomes $\geq \$ 2101$ per month. Patients with a past history of mental disorders had 30.16 higher odds of receiving a diagnosis than patients without past mental disorder histories $(\mathrm{OR}=30.20$, CI $[18.67,48.72])$. All covariates except less than high school education attainment and monthly household income of \$301$\$ 900$ remained significant after adjusting for multiple comparisons. 
Table 4 Adjusted multinomial logistic regression

\begin{tabular}{|c|c|c|c|c|c|c|c|}
\hline & \multirow[t]{2}{*}{ B } & \multirow[t]{2}{*}{ S.E } & \multirow[t]{2}{*}{ Wald $x^{2}$} & \multirow[t]{2}{*}{$p$} & \multirow[t]{2}{*}{ OR } & \multicolumn{2}{|c|}{$\begin{array}{l}95 \% \text { Confidence } \\
\text { interval }\end{array}$} \\
\hline & & & & & & Upper & Lower \\
\hline \multicolumn{8}{|l|}{ Major depressive disorders } \\
\hline Intercept & -1.724 & 0.211 & 66.951 & $<0.001$ & & & \\
\hline Age (years) & -0.010 & 0.003 & 14.326 & $<0.001$ & 0.99 & 0.98 & 0.99 \\
\hline Female $^{a}$ & -0.263 & 0.086 & 9.431 & 0.002 & 0.76 & 0.65 & 0.909 \\
\hline Unmarried $^{b}$ & 0.346 & 0.080 & 18.617 & $<0.001$ & 1.41 & 1.20 & 1.65 \\
\hline Not employed ${ }^{c}$ & 0.426 & 0.117 & 13.331 & $<0.001$ & 1.53 & 1.21 & 1.92 \\
\hline No formal education ${ }^{d}$ & 0.141 & 0.316 & 0.199 & 0.65 & 1.15 & 0.62 & 2.13 \\
\hline$<$ High school $^{d}$ & 0.098 & 0.111 & 0.783 & 0.37 & 1.10 & 0.88 & 1.37 \\
\hline High school ${ }^{d}$ & 0.069 & 0.092 & 0.562 & 0.453 & 1.07 & 0.89 & 1.28 \\
\hline$\leq \$ 300^{e}$ & 0.755 & 0.165 & 20.912 & $<0.001$ & 2.12 & 1.53 & 2.94 \\
\hline$\$ 301-\$ 900^{e}$ & 0.313 & 0.140 & 4.964 & 0.02 & 1.36 & 1.03 & 1.79 \\
\hline$\$ 901-\$ 1500^{e}$ & 0.211 & 0.142 & 2.209 & 0.13 & 1.23 & 0.93 & 1.63 \\
\hline$\$ 1501-\$ 2100^{e}$ & -0.025 & 0.169 & 0.022 & 0.88 & 0.97 & 0.70 & 1.35 \\
\hline Previous mental disorder & 2.032 & 0.082 & 620.783 & $<0.001$ & 7.62 & 6.50 & 8.95 \\
\hline Substance use/abuse history ${ }^{g}$ & 0.211 & 0.092 & 5.248 & 0.02 & 1.23 & 1.03 & 1.48 \\
\hline \multicolumn{8}{|l|}{ Neurocognitive disorders } \\
\hline Intercept & -5.177 & 0.279 & 344.717 & $<0.001$ & & & \\
\hline Age (years) & 0.056 & 0.003 & 259.377 & $<0.001$ & 1.051 & 1.058 & 1.065 \\
\hline Female $\mathrm{a}^{\mathrm{a}}$ & -0.601 & 0.086 & 49.125 & $<0.001$ & 0.54 & 0.46 & 0.64 \\
\hline Unmarried $^{b}$ & 0.239 & 0.082 & 8.504 & 0.004 & 1.27 & 1.08 & 1.49 \\
\hline Not employed ${ }^{c}$ & 0.247 & 0.145 & 2.886 & 0.08 & 1.28 & 0.96 & 1.70 \\
\hline No formal education ${ }^{d}$ & 0.162 & 0.275 & 0.349 & 0.55 & 1.17 & 0.68 & 2.01 \\
\hline$<$ High school $^{d}$ & 0.134 & 0.108 & 1.544 & 0.21 & 1.14 & 0.92 & 1.41 \\
\hline High school $^{d}$ & 0.103 & 0.099 & 1.086 & 0.29 & 1.10 & 0.91 & 1.34 \\
\hline$\leq \$ 300^{e}$ & 0.563 & 0.174 & 10.511 & 0.001 & 1.75 & 1.25 & 2.46 \\
\hline$\$ 301-\$ 900^{e}$ & 0.240 & 0.144 & 2.791 & 0.09 & 1.27 & 0.95 & 1.68 \\
\hline$\$ 901-\$ 1500^{e}$ & 0.100 & 0.146 & 0.470 & 0.49 & 1.10 & 0.83 & 1.47 \\
\hline$\$ 1501-\$ 2100$ & -0.028 & 0.173 & 0.027 & 0.87 & 0.97 & 0.69 & 1.36 \\
\hline Previous mental disorder & 0.646 & 0.098 & 43.326 & $<0.001$ & 1.90 & 1.57 & 2.31 \\
\hline Substance use/abuse history ${ }^{9}$ & -0.070 & 0.098 & 0.510 & 0.47 & 0.933 & 0.770 & 1.12 \\
\hline \multicolumn{8}{|l|}{ Anxiety disorders } \\
\hline Intercept & -1.899 & 0.313 & 36.860 & $<0.001$ & & & \\
\hline Age (years) & -0.012 & 0.004 & 8.235 & 0.004 & 0.989 & 0.981 & 0.997 \\
\hline Female $^{\mathrm{a}}$ & -0.088 & 0.138 & 0.410 & 0.52 & 0.91 & 0.69 & 1.19 \\
\hline Unmarried $^{b}$ & 0.055 & 0.127 & 0.189 & 0.66 & 1.05 & 0.82 & 1.35 \\
\hline Not employed ${ }^{c}$ & -0.209 & 0.162 & 1.654 & 0.19 & 0.81 & 0.59 & 1.11 \\
\hline No formal education ${ }^{d}$ & 0.053 & 0.544 & 0.010 & 0.92 & 1.05 & 0.36 & 3.06 \\
\hline$<$ High schoold $^{d}$ & 0.052 & 0.182 & 0.082 & 0.77 & 1.05 & 0.73 & 1.50 \\
\hline High schoold & 0.026 & 0.145 & 0.032 & 0.85 & 1.02 & 0.77 & 1.36 \\
\hline$\leq \$ 300^{e}$ & 0.051 & 0.277 & 0.034 & 0.85 & 1.05 & 0.61 & 1.81 \\
\hline$\$ 301-\$ 900^{e}$ & 0.304 & 0.208 & 2.131 & 0.14 & 1.35 & 0.90 & 2.03 \\
\hline$\$ 901-\$ 1500^{e}$ & 0.033 & 0.214 & 0.023 & 0.87 & 1.03 & 0.67 & 1.57 \\
\hline$\$ 1501-\$ 2100^{e}$ & -0.048 & 0.250 & 0.037 & 0.84 & 0.95 & 0.58 & 1.55 \\
\hline Previous mental disorder ${ }^{f}$ & 1.495 & 0.127 & 138.713 & $<0.001$ & 4.46 & 3.47 & 5.72 \\
\hline Substance use/abuse history ${ }^{9}$ & 0.154 & 0.148 & 1.083 & 0.29 & 1.16 & 0.873 & 1.55 \\
\hline \multicolumn{8}{|l|}{ Substance-related disorders } \\
\hline Intercept & -3.447 & 0.490 & 49.526 & $<0.001$ & & & \\
\hline
\end{tabular}


Table 4 (continued)

\begin{tabular}{|c|c|c|c|c|c|c|c|}
\hline & \multirow[t]{2}{*}{ B } & \multirow[t]{2}{*}{ S.E } & \multirow[t]{2}{*}{ Wald $x^{2}$} & \multirow[t]{2}{*}{$p$} & \multirow[t]{2}{*}{ OR } & \multicolumn{2}{|c|}{$\begin{array}{l}95 \% \text { Confidence } \\
\text { interval }\end{array}$} \\
\hline & & & & & & Upper & Lower \\
\hline Age (years) & -0.029 & 0.005 & 31.886 & $<0.001$ & 0.97 & 0.96 & 0.98 \\
\hline Female ${ }^{a}$ & -1.317 & 0.197 & 44.755 & $<0.001$ & 0.26 & 0.18 & 0.39 \\
\hline Unmarried $^{b}$ & 0.643 & 0.176 & 13.280 & $<0.001$ & 1.90 & 1.34 & 2.68 \\
\hline Not employed ${ }^{c}$ & -0.001 & 0.204 & 0.000 & 0.99 & 0.99 & 0.67 & 1.49 \\
\hline No formal Education ${ }^{d}$ & -0.279 & 0.793 & 0.124 & 0.72 & 0.75 & 0.16 & 3.57 \\
\hline$<$ High school $^{d}$ & 0.356 & 0.235 & 2.294 & 0.13 & 1.42 & 0.90 & 2.26 \\
\hline High school ${ }^{d}$ & 0.387 & 0.197 & 3.879 & 0.04 & 1.47 & 1.00 & 2.16 \\
\hline$\leq \$ 300^{e}$ & 1.016 & 0.346 & 8.605 & 0.003 & 2.76 & 1.40 & 5.44 \\
\hline$\$ 301-\$ 900^{e}$ & 0.584 & 0.314 & 3.471 & 0.06 & 1.79 & 0.97 & 3.31 \\
\hline$\$ 901-\$ 1500^{e}$ & 0.417 & 0.326 & 1.631 & 0.20 & 1.51 & 0.80 & 2.87 \\
\hline$\$ 1501-\$ 2100^{e}$ & 0.396 & 0.373 & 1.123 & 0.28 & 1.48 & 0.71 & 3.08 \\
\hline Previous mental disorder ${ }^{f}$ & 1.053 & 0.167 & 39.982 & $<0.001$ & 2.86 & 2.06 & 3.97 \\
\hline Substance use/abuse history ${ }^{9}$ & 2.832 & 0.246 & 132.657 & $<0.001$ & 16.98 & 10.48 & 27.49 \\
\hline \multicolumn{8}{|l|}{ Schizophrenia spectrum disorders } \\
\hline Intercept & -5.054 & 0.598 & 71.347 & $<0.001$ & & & \\
\hline Age (years) & -0.028 & 0.005 & 27.363 & $<0.001$ & 0.97 & 0.96 & 0.98 \\
\hline Female $^{\mathrm{a}}$ & -0.929 & 0.181 & 26.251 & $<0.001$ & 0.39 & 0.27 & 0.56 \\
\hline Unmarried $^{b}$ & 0.914 & 0.205 & 19.835 & $<0.001$ & 2.49 & 1.66 & 3.73 \\
\hline Not employed ${ }^{c}$ & 0.959 & 0.311 & 9.510 & 0.002 & 2.60 & 1.41 & 4.79 \\
\hline No formal Education $^{d}$ & 0.512 & 0.781 & 0.430 & 0.51 & 1.66 & 0.36 & 7.71 \\
\hline$<$ High schoold $^{d}$ & 0.758 & 0.248 & 9.331 & 0.002 & 2.13 & 1.31 & 3.47 \\
\hline High schoold & 0.400 & 0.213 & 3.531 & 0.06 & 1.49 & 0.98 & 2.26 \\
\hline$\leq \$ 300^{\mathrm{e}}$ & 1.454 & 0.444 & 10.722 & 0.001 & 4.28 & 1.79 & 10.22 \\
\hline$\$ 301-\$ 90^{e}$ & 1.107 & 0.419 & 6.983 & 0.008 & 3.02 & 1.33 & 6.87 \\
\hline$\$ 901-\$ 1500^{e}$ & 0.382 & 0.444 & 0.738 & 0.39 & 1.46 & 0.61 & 3.49 \\
\hline$\$ 1501-\$ 2100^{e}$ & 0.090 & 0.540 & 0.028 & 0.86 & 1.09 & 0.38 & 3.15 \\
\hline Previous mental disorder ${ }^{f}$ & 3.407 & 0.245 & 194.018 & $<0.001$ & 30.16 & 18.67 & 48.72 \\
\hline Substance use/abuse history ${ }^{g}$ & 0.032 & 0.189 & 0.029 & 0.86 & 1.033 & 0.712 & 1.49 \\
\hline
\end{tabular}

Reference category for DSM-5 diagnoses is no current DSM-5 mental disorders

${ }^{\text {a }}$ Male is reference category

${ }^{\mathrm{b}}$ Married is reference category

'Employed is reference category

$\mathrm{d}$ Postsecondary education and beyond is reference category

e $\geq \$ 2101$ monthly household income is reference category

${ }^{f}$ No previous mental disorder history is reference category

${ }^{9}$ Negative substance use/abuse history is reference category

* $\mathrm{p}$-values reported are those obtained before the Benjamini-Hochberg procedure was applied

\section{Discussion}

After controlling for covariates in the multinomial model, spurious associations were discovered among anxiety, substance-related, and schizophrenia spectrum disorders. Covariates of major depressive and neurocognitive disorders, however, not only maintained significance, but increased in odds ratio from the unadjusted model. Thus, the following discussion will highlight select implications regarding major depressive and neurocognitive disorders diagnosed in inpatients receiving medical or surgical care in a general hospital. Overall, $53 \%$ of our sample of medical inpatients received a current DSM-5 mental disorder diagnosis. While seemingly high, it is comparable to other studies that have characterized mental illness prevalence among medical inpatients $[5,18,33,34,46]$. 


\section{Major depressive disorders}

Major depressive disorder (MDD) is a mood disorder that negatively impacts affective, cognitive, physiological, and behavioral systems. Hallmark features of MDD are sadness, inability to enjoy things that were once pleasurable, and a myriad of signs and symptoms ranging from lack difficulty concentrating and insomnia, to fluctuation in weight and changes in eating patterns. In the severest form, patients with MDD may evidence recurring thoughts of death, suicidal ideation, and even suicide attempts [3]. A recent epidemiological study revealed that among non-institutionalized community members, MDD was slightly higher in Puerto Rican adults living on the island (9.9\%) compared to the U.S. adult population (8.5\%) [10]. Traditionally, among U.S. adults, MDD rates are higher in females compared to males, $8.7 \%$ and $5.3 \%$, respectively, and is most prevalent in people aged 18 to 25. [26]. Sociodemographic factors such as low educational attainment, economic instability, and lack of social support are implicated in the etiology and course of MDD [4].

Traditionally, females are disproportionally affected by $\operatorname{MDD}[2,23,26,28]$. However, this was not the case in this sample of general hospital inpatients. Male patients evidenced 1.30 higher odds of being diagnosed with a major depressive disorder compared to females. At least two plausible explanations can account for this finding. First, in this setting where evaluations and diagnoses were made (a general hospital), both males and females were equally likely to have received a psychological evaluation and subsequent diagnosis, if warranted. Yet, this is not the case in community and outpatient settings, where females are more likely to seek mental healthcare services than males $[25,36,38]$. It begs the question if higher MDD prevalence rates among women are a function of healthcare-seeking behavior or due to actual biological sex-related difference in development and course of depression. Secondly, considering that male patients evidenced higher proportions of mental and physical illness comorbidities than females, $59 \%$ and $48 \%$, respectively, it is plausible that males engaged in relatively maladaptive coping mechanisms in managing their conditions, contributing to depressive affect and subsequent diagnosis during hospitalization. Nevertheless, this does not fully explain why male patients evidenced higher odds of being diagnosed with MDD during hospitalization compared to females.

Regarding the associations between sociodemographic variables spanning age, civil status, employment status, income level and a current diagnosis of depression among this present study's sample, our findings are consistent with existing literature describing non-medical and medical populations [1, 4, 27, 32, 33, 46]. In short, relatively older age and being married is associated with a lower incidence of receiving a current depression diagnosis. Unemployment (including retirement), and lower income is associated with higher incidence of receiving a current depression diagnosis (see Additional file 1).

\section{Neurocognitive disorders}

Neurocognitive Disorders (NCD) are a group of disorders that are characterized by significant clinical dysfunction in areas of complex attention, executive functioning, perpetual motor skills, social cognition, and emotional reaction. Such dysfunction is readily recognizable from premorbid functioning and unfolds in a progressive manner [3]. Considering there are 10 million new cases of NCDs registered yearly, it is estimated that by 2030 there will be an alarming 82 million people suffering from a form of NCD worldwide [47]. NCDs have been among the most prevalent causes of death in the world. While much less is known about the prevalence rates of NCD in Puerto Rico, researchers have estimated that $5 \%$ of adults who are 45 years of age and older report cognitive decline. Of those $5 \%$, the vast majority (87\%) of people with subjective cognitive decline have at least one chronic physical condition [11].

In our sample, as expected, relatively older age was associated with receiving a current NCD diagnosis, specifically, a delirium diagnosis during hospitalization, where each one-year increase in age was associated with 1.05 higher odds of receiving an NCD diagnosis. Delirium disorders are common among elderly patients admitted to general hospitals, with estimates ranging from 14 and $56 \%$ of all elderly patients being affected during hospital stay $[3,16]$. Even though most patients that present with delirium fully recover, if not detected and treated promptly, delirium can lead to adverse consequences and even mortality, especially among older patients [19]. Interestingly and fortunately for the patients, in this present study, unlike major depressive disorders, NCDs were diagnosed more owing to the standing order routine evaluations compared to consults requested by attending physicians, suggesting that if it had not been for routine the evaluations in place, rapid identification of an NCD and initiation of appropriate psychosocial interventions would have possibly been forfeited.

In our study, the second most single common NCD was unspecified neurocognitive disorder, accounting for $14.8 \%$ of all NCD diagnoses. In terms of sociodemographic factors, increasing age, especially individuals over 60 years is associated with receiving a current NCD diagnosis [3]. While there are sex-related differences associated with various subtypes of NCDs, generally, females have higher rates of diagnosed NCDs compared to males. This difference is believed to be due to greater longevity 
in females [17]. Our findings differ slightly from existing literature as males were more likely to have received a current NCD diagnosis. In fact, female patients evidenced $45 \%$ lower odds of being diagnosed with an NCD than males. We believe these findings are a function of the healthcare seeking behavior specific to this sample as discussed in the section regarding MDD diagnoses.

\section{Key implications}

The vast majority (90\%) of patients that were diagnosed with MDD and $87 \%$ that were diagnosed with NCD received an evaluation within $24 \mathrm{~h}$ of being admitted to corresponding hospital units or after physician or patient requested consultations. Swift detection of a current mental disorder during hospitalization and initiation of appropriate interventions is largely due to the integration of clinical health psychology services within a general hospital care delivery model. The CPSP is part of a process-based integration model [22]. In a processbased integration model, a set of methods and models are intertwined to ensure the alignment of financial, administrative, organizational, and clinical functions, with the intention of effectively combining the provision of two or more originally separate clinical services (eg. Coronary care and clinical health psychology). When done correctly, process-based integration results in a fluid, interdisciplinary approach that generates benefits for the whole person/patient and can be conceptualized as integrated care [22]. The implementation of process-based comprehensive care models can be located within one or more of the following general typologies: (a) organizational, (b) functional (c) service, and (d) clinical [24]. Organizational integration occurs when two or more separate organizations (for example, a general hospital and a graduate health sciences institution) join forces to some extent to align the delivery of care for a given patient population. Functional integration is the exchange of non-clinical support components, such as electronic medical records, within and between organizations and clinical units. Service integration is most easily identified as a team of professionals offering their perspective clinical services in a comprehensive and interconnected manner. Finally, clinical integration requires the provision of care by an interdisciplinary team through the use of shared guidelines or protocols (for example, a standing administrative order requiring that all patients admitted to the clinical unit undergo a screening) and standardized assessment.

The model implemented by the CPSP incorporates, to some degree, organizational, functional, service, and clinical components. The program has two modalities of patient evaluation (standardized administrative orders and medical referrals/consults). There is organizational integration established through a collaborative agreement between Hospital Damas and Ponce Health Sciences University (the home institution of our clinical and research teams). Also, the CPSP is located in physical space within the hospital which allows for quick response times and direct contact with doctors and nurses. Contact with these health service providers helps in obtaining direct information on the medical conditions of patients, contributes to reaching an accurate diagnosis of mental disorders, and facilitates the implementation of comprehensive treatment. This approach has been shown to help improve health outcomes for patients and reduce costs related to healthcare expenses [12].The theoretical model of integrated care on which CPSP operates was designed and implemented by [20], the CPSP coordinator and senior researcher and author of our research team.

Interventions facilitated by the clinical team were implemented at the patient's bedside during the course of the initial clinical evaluation. Follow-up sessions were scheduled if warranted. Interventions were tailored to meet the patient's most pressing need during hospitalization and spanned cognitive and behavioral techniques primarily aimed at improving cognitive restructuring, emotion regulation, activation of physiologic relaxation response and solution focused techniques to enhance adherence to medical treatment regimens. While not detailed in this present study, the clinical team referred patients to specialty care services when necessary and implemented brief interventions with family and other healthcare workers including physicians, nurses and social workers as needed to enhance the clinical health psychology services provided. Additionally, patients that require out-patient behavioral health services (e.g., psychotherapy and pharmacotherapy) are referred to our community health center (Wellness Center of Ponce Health Sciences University).

\section{Strengths, limitations, and future work}

While the results from this study cannot support a causative link between sociodemographic factors, previous psychiatric history, and current mental disorder diagnosis, the relatively large sample size and applied research approach allow these findings to be generalized outside of this sample of patients. A possible limitation for this study, however, is the research team did not analyze quantitative psychological symptom inventories (e.g., BDI-II) as covariates. These inventories are administered at patient's bedside to aid in arriving at an accurate current diagnosis. However, these inventories were not available in the digital database as were the other variables analyzed in this study. Also, our clinical team did not implement measures to initiate patient follow-up post hospital discharge for symptom monitoring or continued 
psychotherapy. Due to the brief (average two-to-three sessions) and often time single session encounters that is common in C-L psychology in general hospital settings, it is challenging to gauge the effectiveness of the treatment interventions once patients are discharged. This is concerning as the ultimate goal of intervening in medical patients is to enhance overall health outcomes by addressing pressing mental health challenges that may be interfering with physical health and daily functioning. When there is no follow-up system in place, there is no way of knowing if patients actually benefited from the interventions of if they require more long-term therapy. There is limited literature detailing the effectiveness of post-discharge C-L follow-up systems in identifying areas of patient need and continued therapy $[13,35]$. Thus, future clinical and research work related to designing and implementing post-discharge follow-up is a promising avenue.

\section{Conclusion}

Major depressive, neurocognitive, anxiety, substance use, and schizophrenia-spectrum disorders were the five most commonly made DSM-5 diagnoses in a general hospital system. Male patients were 1.30 times more likely to have been diagnosed with major depressive disorder compared to females, and delirium was the most common form of NCD among both males and females. The importance of integrating clinical health psychology services within the administrative and clinical structure of general hospitals was discussed. Future work concerning the opportunities, barriers and program evaluation methods are needed. Additionally, ways of continuing and or monitoring of psychological care post discharge is worth future clinical and research efforts.

\section{Supplementary Information}

The online version contains supplementary material available at https://doi. org/10.1186/s13033-021-00484-w.

Additional file 1. Commonly Diagnosed Mental Disorders in a General Hospital System.

\section{Acknowledgements}

The work presented was supported by the Research Centers for Minority Institutions (RCMI-U54MD007579).

\section{Authors' contributions}

Supervision of clinical team: JG.H-J, AR-L; data analysis: GS, Gloria A-T; manuscript Preparation: GS, AM.B-L, AA.R-J and JJ-C. All authors have read and approved the final manuscript.

\section{Funding}

Dr. Julio Jiménez has received a research grant from the Research Centers for Minority Institute (RCMI-U54MD007579).
Availability of data and materials

Not applicable.

\section{Declarations}

\section{Ethics approval and consent to participate}

All procedures performed in this study were in accordance with the ethical standards of the Ethics Committee of the Ponce Research Institute and adhere to the tenants of the 1964 Helsinki Declaration and its later amendments. Informed consent was obtained from all individual participants included in the study.

\section{Consent to publication}

All participants signed informed consent regarding publishing their data.

\section{Competing interests}

All other authors declare they have no conflict of interest.

\section{Author details}

${ }^{1}$ Health Psychology Training and Research Core, School of Behavior and Brain Sciences, Ponce Health Sciences University, 388 Zona Industrial Reparada 2, Ponce, PR 00717, USA. ${ }^{2}$ Department of Behavioral Medicine, Damas Hospital, 2213 Ponce Bypass, Ponce, PR 00717, USA.

Received: 7 November 2020 Accepted: 3 June 2021

Published online: 19 June 2021

\section{References}

1. Akhtar-Danesh N, Landeen J. Relation between depression and sociodemographic factors. Int J Ment Health Syst. 2007;1 (1):4. https://doi.org/10. 1186/1752-4458-1-4.

2. Albert PR. Why is depression more prevalent in women? I Psychiatry Neurosci. 2015:40(4):219-21. https://doi.org/10.1503/jpn.150205.

3. American Psychiatric Association. Diagnostic and statistical manual of mental disorders (5th ed.). American Psychiatric Association. 2019.

4. Assari S, Lankarani MM. Reciprocal associations between depressive symptoms and mastery among older adults: black-white differences. Front Aging Neurosci. 2017. https://doi.org/10.3389/fnagi.2016.00279.

5. Al-Atram AA. Prevalence and patterns of psychiatric co-morbidity among adult medical inpatients: a cross-sectional study. Kuwait Med J. 2018;50(4):410-6.

6. Beck AT, Steer RA, Brown GK. Beck depression inventory manual. 2nd ed. San Antonio: Psychological corporation; 1996.

7. Beck AT, Epstein N, Brown G, Steer RA. An inventory for measuring clinical anxiety: psychometric properties. J Consult Clin Psychol. 1988;56(6):893-7.

8. Benjamini Y, Hochberg Y. Controlling the false discovery rate: a practical and powerful approach to multiple testing. J R Stat Soc Series B Stat Methodol. 1995;57(1):289-300.

9. Canino G, Vila D, Santiago-Batista K, García P, Velez-Baez G, MoredaAlegría A. Needs assessment study of mental health and substance use disorders and service utilization among adult population of Puerto Rico. ASSMCA. (2016) https://www.assmca.pr.gov. Accessed 5 Nov 2020.

10. Canino G, Shrout PE, NeMoyer A, Vila D, Santiago KM, Garcia P, Quiñones A, Cruz V, Alegria M. A comparison of the prevalence of psychiatric disorders in Puerto Rico wiht the United States and Puerto Rican population of the United States. Soc Psychiatry Psychiatric Epidemiol. 2019;54:369-78,

11. Center for Disease Control and Prevention. Subjective cognitive decline Puerto Rico. (2019)https://www.cdc.gov/aging/data/infographic/2017/ puerto-rico-cognitive-decline.html. Accessed 2 Nov 2020.

12. Epstein NE. Multidisciplinary in-hospital teams improve patient outcomes: a review. Surg Neurol Int. 2014;5(Suppl 7):S295-303. https://doi. org/10.4103/2152-7806.139612.

13. Ehrenreich MJ, Robinson CT, Glovinsky DB, Dixon LB, Medoff DR, Himelhoch SS. Medical inpatients' adherence to outpatient psychiatric aftercare: a prospective Study of patients evaluated by an inpatient consultation liaison psychiatry service. Int J Psychiatry Med. 2012;44(1):1-15. https://doi.org/10.2190/PM.44.1.a. 
14. Fava GA. Psychosomatic medicine. In: Fink G, editor. Stress: Concepts, cognition, emotion and behavior. Cambridge: Academic press; 2016.

15. Folstein MF, Folstein SE, McHugh PR. Mini-mental state: a practical method for grading the cognitive state of patients for the clinician. J Psychiatry Res. 1975;12(3):189-98. https://doi.org/10.1016/0022-3956(75) 90026-6.

16. Fong TG, Tulebaev SR, Inouye SK. Delirium in elder adults: diagnosis, prevention and treatment. Nat Rev Neurol. 2009;5:210-20. https://doi.org/10. 1038/nrneurol.2009.24.

17. Ganguli M. Epidemiology of dementia. In: Abou-Saleh MT, Katona CLE, Kumar A, editors. Principles and practices of geriatric psychiatry. 3rd ed. Hoboken: Wiley; 2012.

18. Hansen MS, Fink P, Frydenberg M, Oxhøj M, Søndergaard L, MunkJørgensen P. Mental disorders among internal medicine inpatients: Prevalence, detection and treatment treatment status. J Psychosom Res. 2001;50(4):199-204. https://doi.org/10.1016/s0222-3999(00)00230-0.

19. Inouye SK. Delirium in older persons. N Engl J Med. 2006;354(11):1157-65.

20. Jiménez J, Rivera D, Benítez P, Tarrats H, Ramos A. Integrating mental health services into a general hospital in Puerto Rico. J Clin Psychol Med Settings. 2013;20(3):294-301. https://doi.org/10.1007/s10880-012-9352-x.

21. Knight M. Access to mental health care among older adults. J Gerontol Nurs. 2011;37(3):16-21.

22. Konder DL, Spreeuwenberg C. Integrated care: meaning, logic applications and implications_a discussion paper. Int J Integr Care. 2(4). 2002 https://www.ijic.org/article/. https://doi.org/10.5334/ijic.67

23. Labaka A, Goñi-Balentziaga O, Lebeña A, Pérez-Tejada J. Biological sex differences in depression: a systematic review. Biol Res Nurs. 2018;20(4):38392. https://doi.org/10.1177/1099800418776082.

24. Lewis R, Rosen R, Goodwin N, Dixon J. Where next for integrated care organizations in the English NHS? The King's Fund. London: The Nuffield Trust; 2010.

25. Menbreu M, Mekonen T, Azale T, Auyano G, Yimer S, Getnet A, Belete A, Kerie S, Fekadu W. Health care seeking behavior for depression in Northeast Ethiopia: depression is not considered as illness by more than half of the participants. Ann Gen Psychiatry. 2018. https://doi.org/10.1186/ s12991-018-0205-3.

26. National Institutes of Mental Health. Depression. 2018 https://www.nimh. nih.gov/health/topics/depression/index.shtml. Accessed 3 Nov 2020.

27. $\mathrm{Ng} \mathrm{CW}, \mathrm{How} \mathrm{CH}, \mathrm{Ng}$ YP. Major depression in primary care: making the diagnosis. Smedj. 2016;57(11):591-7. https://doi.org/10.11622/smedj. 2016174.

28. Picco L, Subramaniam M, Abdin E, Vaingankar JA, Chong SA. Gender difference in major depressive disorder: Findings from the Singapore mental health study. Smedj. 2017;58(11):649-55. https://doi.org/10.11622/smedj. 2016144.

29. Ritchie H, Roser M. Mental health. OurWorldlnData.org. 2018 https:// www.ourworlindata.org/mental-health. Accessed 29 Oct 2020.

30. Rothenhäusler HB. Mental disorders in general hospital patients. Psychiatr Danub. 2006;3(4):183-92.

31. Salgado CM, Azevedo C, Proença H, Vieira SM. Missing data. In: Salgado CM, editor. Secondary Analysis of Electronic Health Records. Cham: Springer; 2016.

32. Sareen J, Afifi TO, McMillan KA, Asmundson GJG. Relationship between household income and mental disorders. Arch Gen Psychiatry. 2011;68(4):419-27.

33. Scott G, Ramos-Pérez N, Ramos-Estremera N, Mirles-Muñiz I, PadillaDiffoot A, Ramos-Lucca A, Asencio-Toro G, Jiménez-Chávez J. Relating sociodemographic factors and glycemic control among inpatients with type 2 diabetes. J Public Health. 2020. https://doi.org/10.1007/ s10389-020-01303-1.

34. Silverstone PH. Prevalence of psychiatric disorders in medical inpatients. J Nerv Ment Dis. 1996;184(1):43-51. https://doi.org/10.1097/00005053199601000-00008.

35. Sloan EP, Kirsh S. Postdischarge impact of $C-L$ psychiatry treatment in obstrerical Inpatients. ISRN Obsterics and Gynecology. 2011. https://doi. org/10.5402/2011/456012.

36. Smith KL, Matheson Fl, Moineddin R, Dunn JR, Lu H, Cairney J, Glaizer RH. Gender differences in mental health service utilization among respondents reporting depression in a national health survey. Sci Res. 2013. https://doi.org/10.4236/health.2013.510212.

37. Stevens L, Rodin I. Liaison psychiatry. In: Stevens L, Rodin I, editors. psychiatry. 2nd ed. Amsterdam: Elsevier; 2011.

38. Thompson AE, Anisimowicz Y, Midema B, Hogg W, Wodchis WP, AubreBassler K. The influence of ender and other patient characteristics on health care-seeking behaviour: a QUALICOPC study. BMC Fam Pract. 2016. https://doi.org/10.1186/s12875-016-0440-0.

39. Thompson TL, Suddath RL, Edward G. Billings MD: Pioneer of consultation-liaison psychiatry. Psychosomatics. 1987;28(3):153-6.

40. Unützer J, Harbin $\mathrm{H}$, Schoenbaum $\mathrm{M}$, Druss B. The collaborative care model: an approach for integrating physical and mental health care in Medicaid health homes. Center for Health Care Strategies and Mathematica Policy and Research. 2013 https://aims.uw.edu/sites/default/ files/CMSBrief_2013.pdf. Accessed 1 Nov 2020.

41. U.S. Bureau of Labor Statistics. Economy at a glance: Puerto Rico. 2020 https://www.bls.gov/eag/eag.pr.htm. Accessed 28 Dec 2020.

42. U.S. Census Bureau (2019). Quick facts: Puerto Rico. 2019. https://www. census.gov/quickfacts/PR. Accessed 28 Dec 2020.

43. Wainberg ML, Scorza P, Shultz JM, Helpman L, Mootz JJ, Johnson KA, Neira Y, Bradford JE, Oquendo MA, Arbuckle MR. Challenges and opportunities in Global mental health: a research-to-practice perspective. Curr Psychiatry Rep. 2017;19(5):28. https://doi.org/10.1007/s11920-017/ s11920-017-0780-Z

44. Walker ER, McGee RE, Druss BG. Mortality in mental disorders and global disease burden implications: a systematic review and meta-analysis. JAMA Psychiat. 2016;72(4):334-41. https://doi.org/10.1001/jamapsychi atry.2014.2502.

45. Walker J, Burke K, Wanat M, Fisher R, Fielding J, Mulick A, Puntis S, Sharpe J, Esposti MD, Harriss E, Frost C, Sharpe M. The prevalence of depression in general Hospital inpatients: a systematic review and meta-analysis of interview-based studies. Psychol Med. 2018;48(14):2285-98. https://doi. org/10.1017/S0033291718000624.

46. Wang Z, Yang H, Guo Z, Liu B, Geng S. Socio-demographic characteristic and co-occurence of depressive symptoms with chronic diseases among older adults in China: the China longitudinal ageing social survey. BMC Psychiatry. 2019. https://doi.org/10.1186/s12888-019-2305-2.

47. World Health Organization Global action plan on the public health response to dementia 2017-2025. Geneva: World Health Organization; 2017. Licence: CC BY-NC-SA 3.0 IGO.

\section{Publisher's Note}

Springer Nature remains neutral with regard to jurisdictional claims in published maps and institutional affiliations. 